\title{
ИНТЕЛЛЕКТУАЛЬНАЯ СИСТЕМА НЕЙРО-НЕЧЕТКОГО АНАЛИЗА И МНОГОМЕРНОГО ПРОГНОЗИРОВАНИЯ СЛОЖНЫХ ПРОЦЕССОВ
}

\section{INTELLIGENT SYSTEM OF NEURO-FUZZY ANALYSIS AND MULTIDIMENSIONAL FORECASTING OF COMPLEX PROCESSES \\ V. Luferov}

Summary. The article deals with the developed intelligent neuro-fuzzy analysis and multidimensional forecasting of complex processes. The system is designed: first, to analyze the direct and indirect interaction of system and external system factors in the study of complex problem situations; second, to model the system dynamics of complex processes occurring within the framework of problem situations; third, for multidimensional forecasting of complex processes.

The intelligent system is based on a complex of intelligent models, methods and technologies (including original ones) focused on intelligent analysis and modeling of complex processes under uncertainty, including: neuro-fuzzy cognitive models, fuzzy evaluation models, and models of system dynamics. The developed intelligent system was tested on the example of multidimensional forecasting of the state of the urban environment.

Keywords: time series, neuro-fuzzy cognitive temporal models, fuzzy logic, multidimensional forecasting.

\author{
Луферов Виктор Сергеевич \\ Аспирант, ФГБОУ ВО Национальный \\ исследовательский университет «МЭИ» (Московский \\ энергетический институт) \\ lyferov@yandex.ru
}

Аннотация. В статье рассматривается разработанная интеллектуальная нейро-нечеткого анализа и многомерного прогнозирования сложных процессов. Система предназначена: во-первых, для анализа непосредственного и опосредованного взаимовлияния системных и внешних системных факторов при исследовании сложных проблемных ситуаций; в0-вторых, для моделирования системной динамики сложных процессов, происходящих в рамках проблемных ситуаций; в-третьих, для многомерного прогнозирования сложных процессов.

Интеллектуальная система основана на комплексе интеллектуальных моделей, методов и технологий (в том числе оригинальных), ориентированных на интеллектуальный анализ и моделирование сложных процессов в условиях неопределенности, в том числе: нейро-нечетких когнитивных моделей, нечетких оценочных моделей, моделей системной динамики. Апробация разработанной интеллектуальной системы проведена на примере многомерного прогнозирования состояния городской среды.

Ключевые слова: временной ряд, нейро-нечеткие когнитивные темпоральные модели, нечеткая логика, многомерное прогнозирование.

сложных процессов ограничено возможностями моделей динамики в условиях ограниченных данных.

Ранее в работах [5] был предложен метод анализа и прогнозирования многомерных временных рядов (ВР), основанный на предлагаемом типе нейро-нечетких когнитивных темпоральных моделей (ННКТМ), обеспечивающий учет непосредственного, опосредованного и аккумулированного взаимовлияния сложных процессов, а также позволяющий осуществлять их моделирование и прогнозную оценку в условиях неполноты информации, неопределенности, нелинейности взаимовлияния, частичной несогласованности и существенной взаимозависимости как при отсутствии, так и при наличии внешнего воздействия.

В статье рассмотрена интеллектуальная система нейро-нечеткого анализа и многомерного прогнозирования сложных процессов, реализующая предлагаемый метод. 


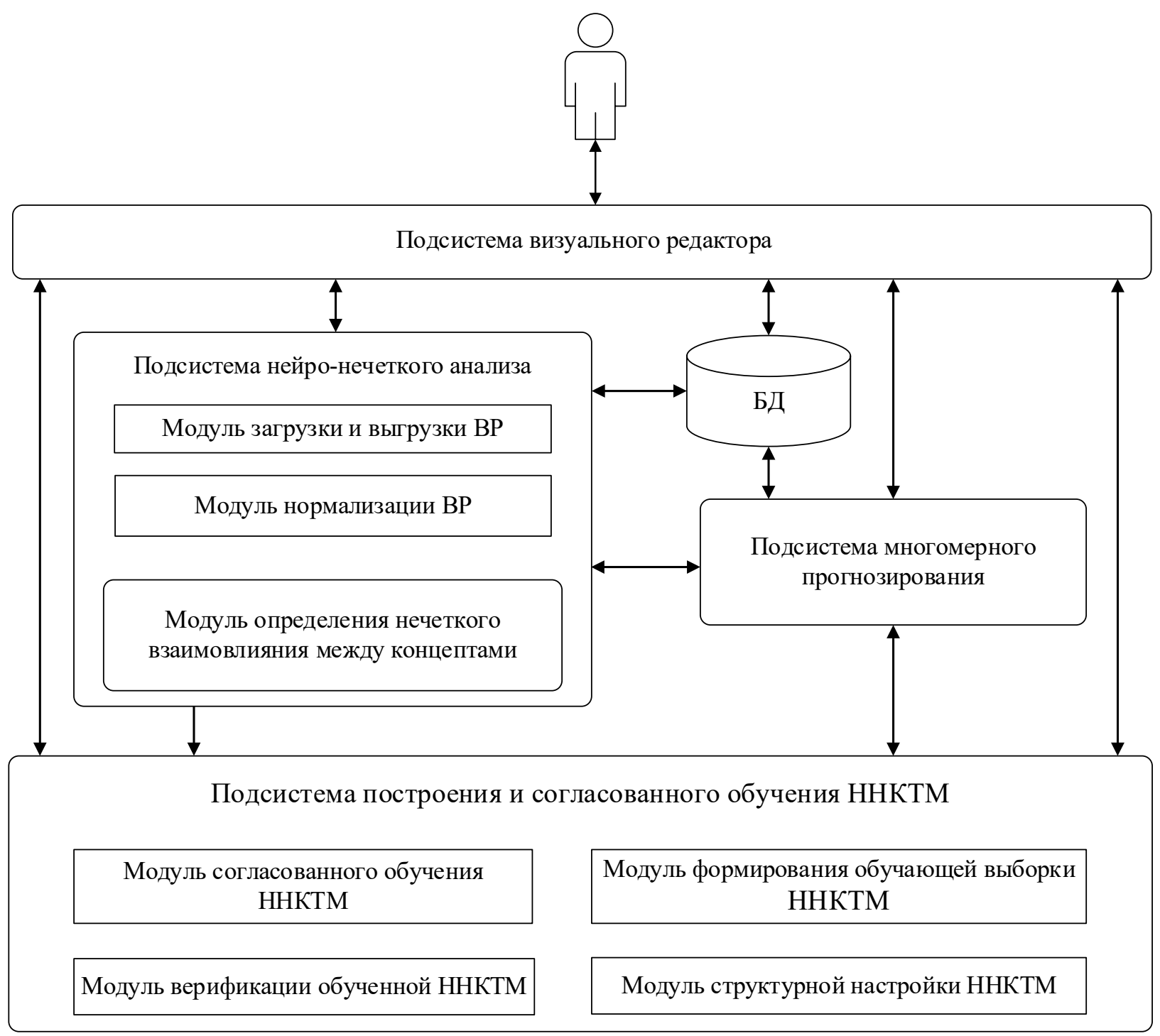

Рис. 1. Модульная структура разрабатываемых программных средств для анализа и прогнозирования MPB на основе НHКTM

\section{Структура разработанной интемлектУа^ьной системы нейро-нечеткого анализа и многомерного прогнозирования сложных прочессов}

На рисунке 1 представлена модульная структура разработанной интеллектуальной системы нейро-нечеткого анализа и многомерного прогнозирования сложных процессов. Рассмотрим кратко назначение отдельных подсистем и модулей разработанной интеллектуальной системы.

Подсистема визуального редактора содержит классы и библиотеки графического интерфейса, отвечающие за корректность вводимых данных, предотвращение ввода неверной информации, а также за пред- ставление пользователю информации в удобном и доступном виде.

Подсистема нейро-нечеткого анализа отвечает за загрузку и выгрузку ВР концептов, выполняет нормализацию ВР. Кроме того, эта подсистема включает в себя модуль определения взаимосвязи между концептами, в результате чего выявляются непосредственные, опосредованные и агрегированные взаимосвязи между концептами, разрешается проблема мультиколлинеарности соответствующих им ВР.

Подсистема многомерного прогнозирования реализует разработанные процедуры: непосредственного прогнозирования ВР концептов по задаваемым совокупностям значений входных переменных; моделирование динамики изменения и прогнозную оценку 


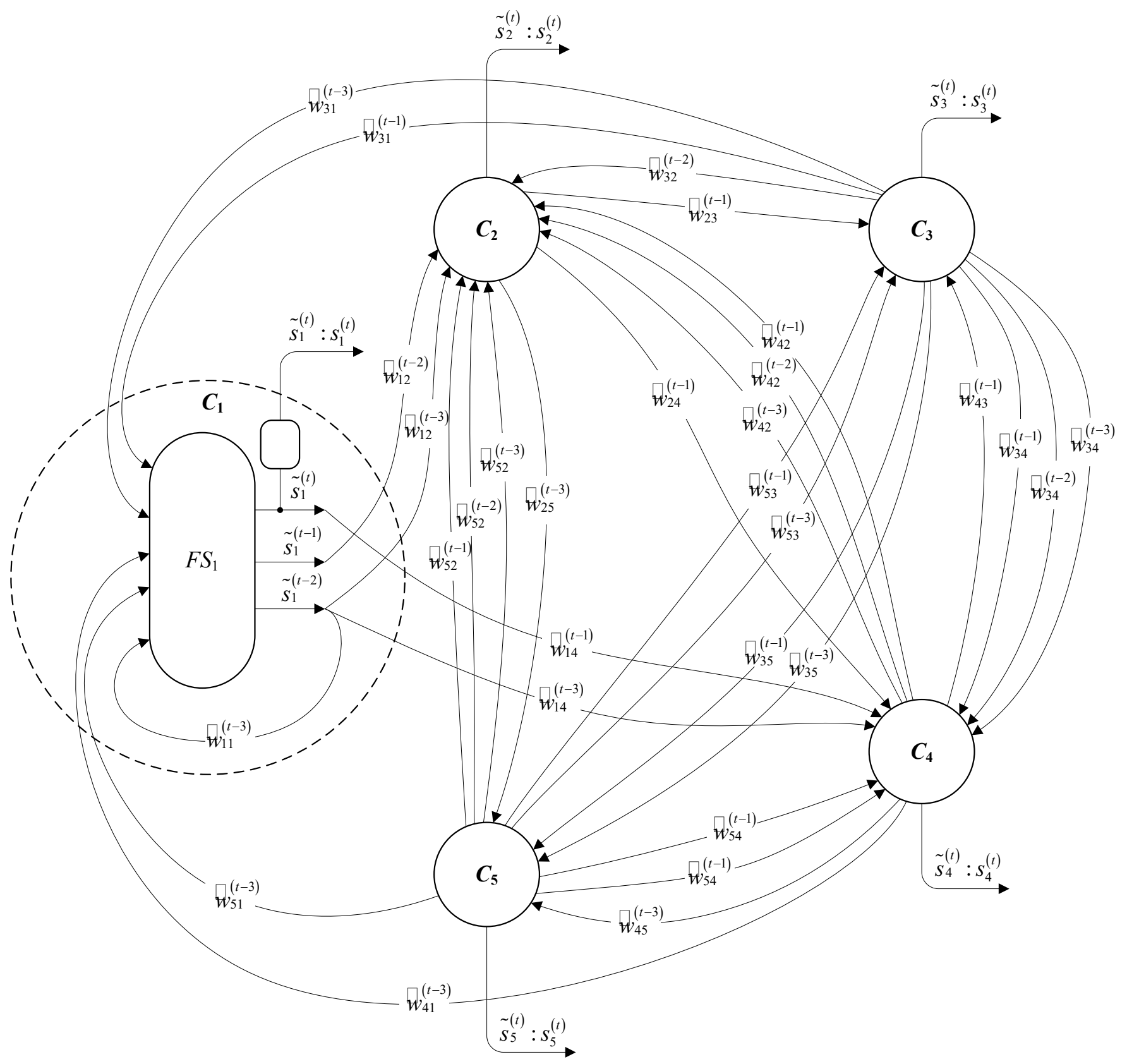

Рис. 2. ННКТМ для прогнозирования состояния городской среды

ВР при отсутствии внешних воздействий; моделирование динамики изменения и прогнозную оценку ВР при внешнем воздействии на значения концептов и/ или на отношения взаимовлияния между концептами HHKTM.

Хранение пользовательских настроек, ВР, нормализованных ВР, параметров моделей ННКТМ осуществляется в оперативной базе данных (БД). Реляционная БД реализует взаимодействие (посредствам $S Q L$ ) с подсистемами визуального редактора, анализа ВР и прогнозирования состояния сложных процессов.
Подсистема построения и согласованного обучения ННКТМ состоит из следующих моделей: формирования обучающей выборки ННКТМ, структурной настройки ННКТМ, согласованного обучения ННКТМ, верификации обученной ННКТМ. Модель структурной настройки ННКТМ выполняет формирование структуры и предварительную параметрическую настройку ННКТМ с учетом непосредственного и опосредованного влияния компонентов МВР друг на друга. Модуль формирования обучающей выборки ННКТМ отвечает за формирование обучающей выборки для согласованного обучения ННКТМ, которое реализуется 


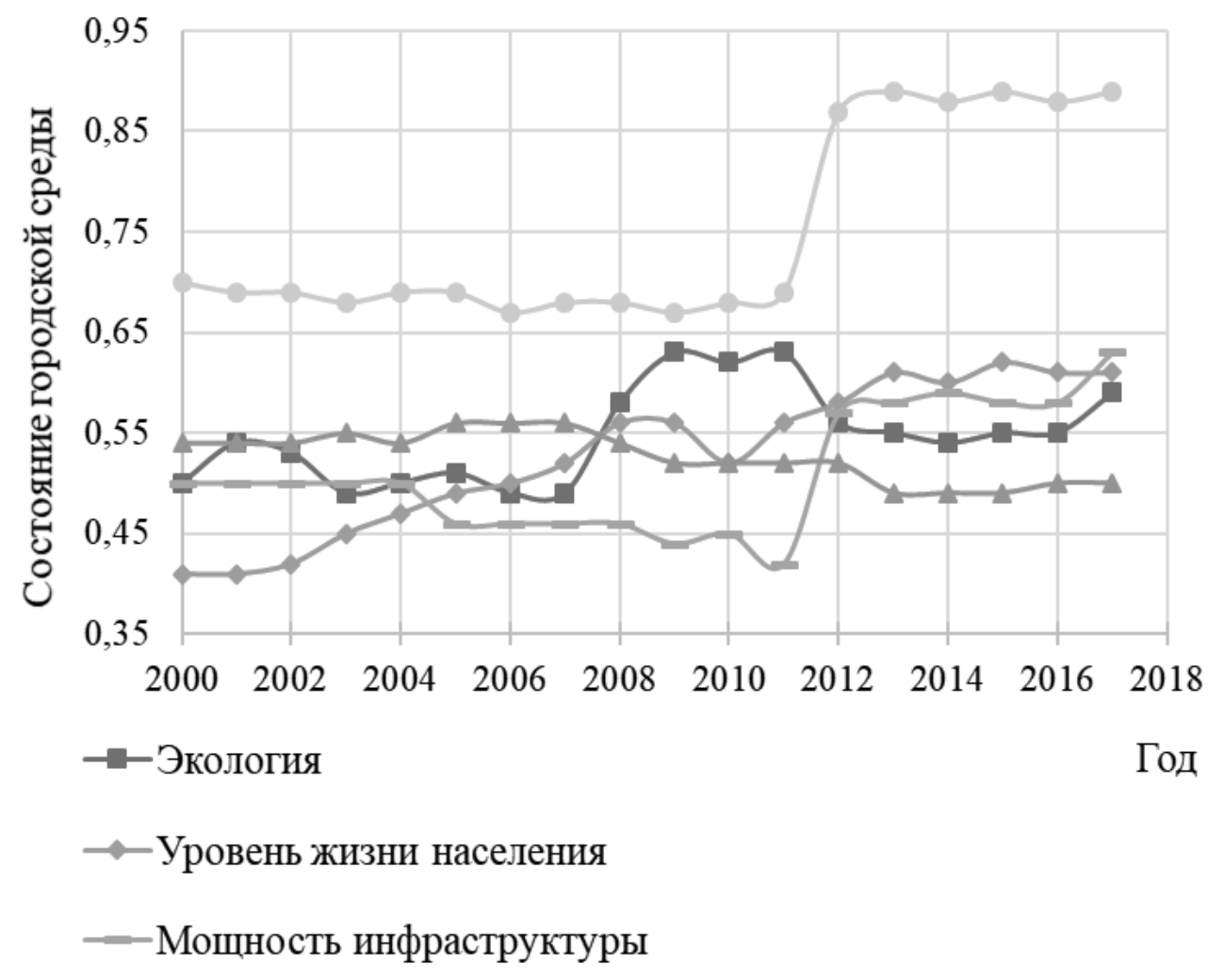

Рис. 3. Прогнозная оценка состояния городской среды г. Москвы

модулем согласованного обучения ННКТМ. Модуль верификации обученной ННКТМ реализует процедуры проверки адекватности модели.

\section{Оченка точности многомерного прогнозирования сАОЖных прочессов с использованием разработанной интемлектуальной системы}

Оценка точности многомерного прогнозирования сложных процессов с использованием разработанной интеллектуальной системы выполнялась на примере оценка состояния городской среды г. Москвы, характеризующейся состоянием ее концептов (объектов, подсистем, характеристик).

В соответствии с этими исследованиями разработана ННКТМ для анализа и многомерного прогнозирования состояния городской среды г. Москвы (рисунок 2).

На основе результатов ранее проведенных исследований [6, 7] определены следующие значимые факторы (компоненты МВP), характеризующие состояние городской среды: $C_{1}$ - экология $(E C O) ; C_{2}$ - мощность инфраструктуры $(I N F) ; C_{3}$ - уровень доходов населения $(U Z N) ; C_{4}$ - промышленное потребление топливно-э- нергетических ресурсов ( $E L O) ; C_{5}$ - качество жизни населения $(K Z N)$.

На рисунке 2: $C$ - множество концептов, определяющих состояние соответствующих концептов городской среды; $\tilde{F}_{i}-$ нечеткое темпоральное преобразование, реализуемое соответствующим концептом $C_{i}$ с учетом нечетких тенденций соответствующего компонента МВР; $N$ - количество концептов ННКТМ;

$\tilde{S}_{i}(t)$ - прогнозируемое нечеткое значение концепта $C_{i}$ в $t$-й момент времени;

$$
\left(\tilde{s}_{j}^{\prime(t-1)}, \ldots, \tilde{s}_{j}^{\prime\left(t-L_{i}^{j}\right)}\right)-
$$

подмножество входных темпоральных нечетких переменных концепта $C_{i}$, связанных с соответствующими выходными темпоральными нечеткими переменными концепта $C_{j}$; $M_{i}$ - число концептов ННКТМ, непосредственно связанных с концептом $C_{i} ; l_{i}^{j}$ - временной лаг (задержка) для соответствующей входной нечеткой темпоральной переменной

$\tilde{s}_{j}^{\prime\left(t-l_{i}^{j}\right)}$ концепта $C_{i}, l_{i}^{j}=0, \ldots, L_{i}^{j} ; W$ - множество отношений непосредственного влияния между всеми парами концептов ННКТМ; $W_{i j}$ - подмножество нечетких значений, определяющее упорядоченный в хронологи- 

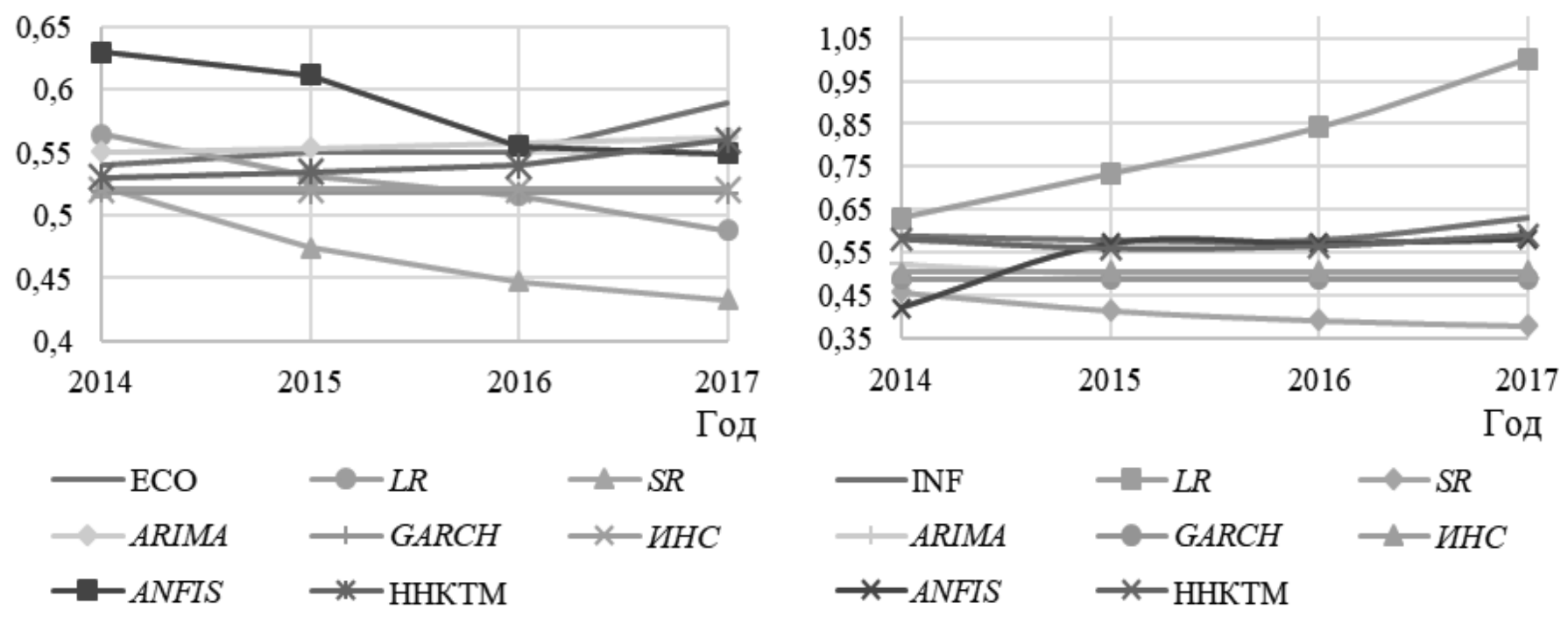

a)

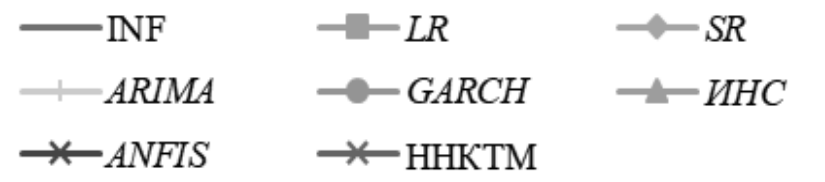

б)
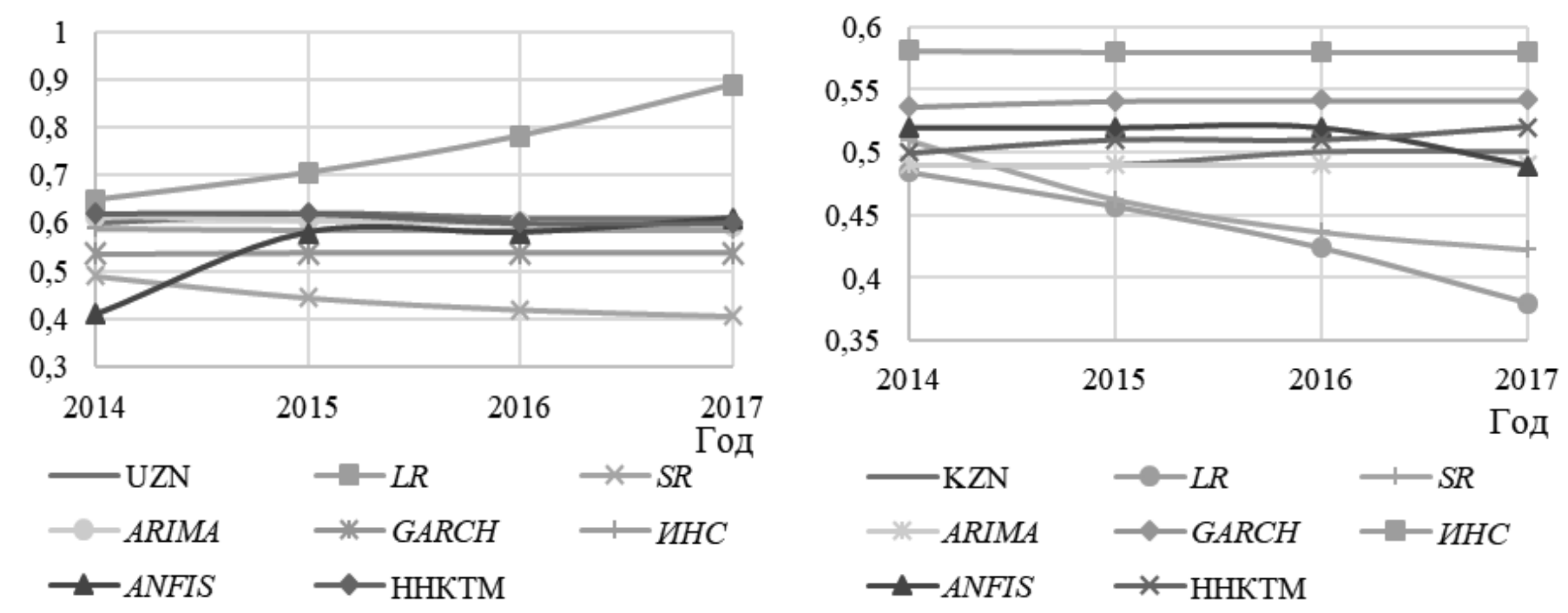

B)

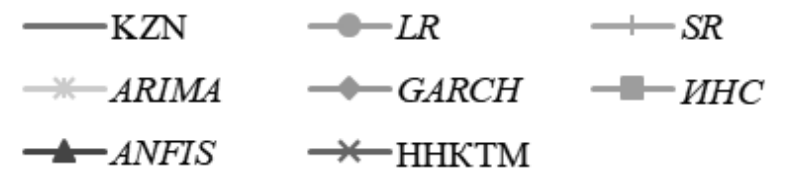

г)
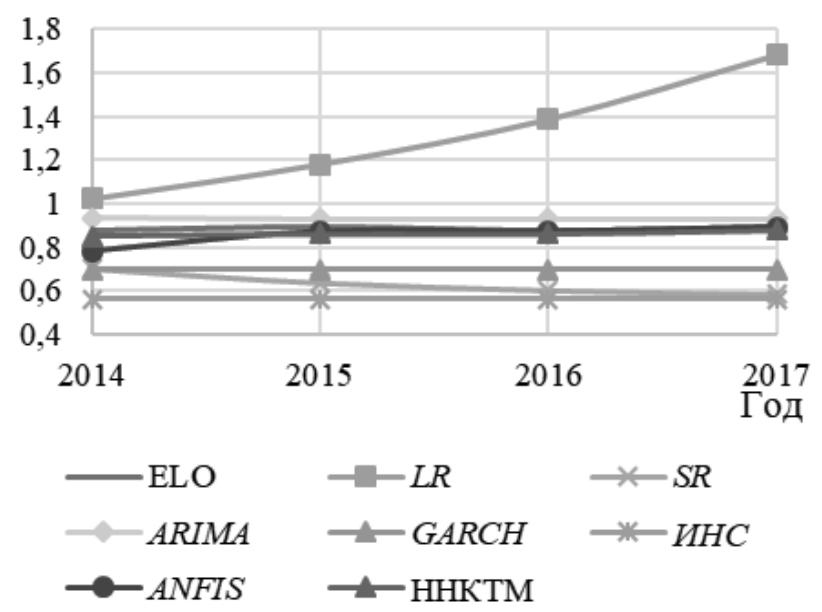

д)

Рис. 4. Иллюстрация результатов многомерного прогнозирования состояния городской среды: а) экология (ECO); б) мощность инфраструктуры (INF); в) уровень доходов населения (UZN); г) качество жизни населения (KZN); д) промышленное потребление топливно-энергетических ресурсов (ELO) 
Таблица 1. Результаты сравнительной оценки многомерного прогнозирования состояния городской среды г. Москвы

\begin{tabular}{|c|c|c|c|c|c|c|c|c|}
\hline \multirow{2}{*}{$\begin{array}{l}\text { № } \\
\Pi / \Pi\end{array}$} & \multirow{2}{*}{ Концепты } & \multicolumn{7}{|c|}{ Точность прогнозирования,\% } \\
\hline & & LR & SR & ARIMA & GARCH & ИНС & ANFIS & HHKTM \\
\hline 1. & Экология & 92,1 & 84,4 & 89,2 & 93,1 & 86,1 & 91,1 & 96,8 \\
\hline 2. & Мощность инфраструктуры & 65,7 & 72,0 & 80,6 & 82,1 & 97,1 & 89,9 & 95,7 \\
\hline 3. & Уровень доходов населения & 76,2 & 69,1 & 83,0 & 88,1 & 98,2 & 89,2 & 97,2 \\
\hline 4. & $\begin{array}{l}\text { Промышленное потребление } \\
\text { топливно-энергетических } \\
\text { ресурсов }\end{array}$ & 51,3 & 71,2 & 81,1 & 78,7 & 78,8 & 96,3 & 96,8 \\
\hline 5. & Качество жизни населения & 88,2 & 90,6 & 90,6 & 90,9 & 85,8 & 95,4 & 96,5 \\
\hline \multicolumn{2}{|c|}{ МВР в целом } & 51,3 & 69,1 & 80,6 & 78,7 & 78,8 & 89,2 & 95,7 \\
\hline
\end{tabular}

ческой последовательности набор нечетких степеней влияния

$\tilde{w}_{i j}^{\left(t-l_{i}^{j}\right)}$ концепта $C_{j}$ на концепт $C_{i}$ с учетом временного лага $l_{i}^{j} ; \tilde{\varphi}_{i j}$ - нечеткий оператор для учета степени взаимовлияния выходной переменной концепта $C_{j}$ на входную переменную концепта $C_{i}$; в случае нечетких переменных в качестве оператора $\tilde{\varphi}_{i j}$ целесообразно использовать Т-норму.

В качестве показателя ошибки прогнозирования выбрана средняя абсолютная процентная ошибка (MAPE - mean absolute percentage error) [8]:

$$
\delta S_{i}=\frac{1}{T} \sum_{t=1}^{T}\left|\frac{S_{i}^{(t)}-S_{i(\mathrm{cur})}^{(t)}}{S_{i}^{(t)}}\right|
$$

где $\delta S_{i}$ - ошибка прогнозирования $i$-го компонента MBP; $S_{i}^{(t)}$ - эталонное значение $i$-го компонента МВР в момент времени $t ; S_{i(c u r)}^{(t)}$ - прогнозное значение $i$-го концепта городской среды в момент времени $t$; $i=1, \ldots, N-$ количество концептов городской среды; $T$ - число прогнозных значений состояния концептов городской среды.

Ошибка прогнозирования состояния городской среды в целом представляется в виде:

$$
\delta S=f\left(\delta S_{1}, \ldots, \delta S_{N}\right)
$$

В данном исследовании прогнозная оценка состояния городской среды г. Москвы оценивалась максимальной ошибкой прогнозного состояния ее концептов:

$$
\delta S=\max \left(\delta S_{1}, \ldots, \delta S_{N}\right) .
$$

Заметим, что исходные данные состояния городской среды изначально приведены в относительных едини- цах. Чем больше значение концепта, тем лучше состояние соответствующего концепта. Временные ряды, характеризующие состояние концептов городской среды г. Москвы для 2000-2017 гг. представлены на рисунке 3. Исходные данные для сравниваемых моделей были разделены на обучающую (80\%) и проверочную (20\%) выборки.

В ходе экспериментов осуществлялась сравнительная оценка точности многомерного прогнозирования состояния городской среды г. Москвы с использованием предлагаемого метода (на основе ННКТМ) по сравнению со следующими моделями: множественная линейная регрессия $(L R)$; линейная регрессия с использованием стохастического градиентного спуска $(S R)$; авторегрессионное интегрированное скользящее среднее (ARIMA); обобщенная авторегрессионная модель гетероскедастичности (GARCH); искусственные нейронные сети прямого распространения сигналов (многослойного персептрона) (ИНС); адаптивная сеть нечеткого вывода (ANFIS).

Результаты сравнительной оценки многомерного прогнозирования состояния городской среды г. Москвы показаны в таблице 1.

Результаты сравнительной оценки показали, что использование предложенной интеллектуальной системы (на основе ННКТМ) позволяет повысить точность многомерного прогнозирования состояния городской среды (на примере г. Москвы) по сравнению с рассмотренными методами, в том числе по сравнению с продемонстрировавшей наилучшие результаты моделью типа ANFIS, в среднем, на 6,5\%.

\section{Зак^ючение}

Разработана интеллектуальная система нейро-нечеткого анализа и многомерного прогнозирования сложных процессов, включающая в себя: подсистему визуального 
редактора; подсистему нейро-нечеткого анализа (состоящую из модулей загрузки и выгрузки ВР, нормализации $\mathrm{BP}$, определения нечеткого взаимовлияния между концептами); оперативную базу данных (для хранения ВP, нормализованных ВР, обученных ННКТМ, параметров обучения и моделирования); подсистему многомерного прогнозирования на основе ННКТМ; подсистему построения и согласованного обучения ННКТМ (состоящую из модулей формирования обучающей выборки ННКТМ, структурной настройки ННКТМ, согласованного обучения ННКТМ, верификации обученной ННКТМ).

Разработана библиотека программных функций, предназначенная для создания и обучения нейро-нечетких моделей, используемых с целью нейро-нечет- кого анализа и многомерного прогнозирования МВР на основе ННКТМ. Библиотека обеспечивает гибкие возможности модульного встраивания в существующие информационно-аналитические и управляющие системы.

Результаты сравнительной оценки показали, что использование разработанной интеллектуальной системы позволяет повысить качество анализа и точность многомерного прогнозирования состояния сложных процессов по сравнению с методами в условиях в условиях неполноты информации, неопределенности, нелинейности взаимовлияния, частичной несогласованности и существенной взаимозависимости системных и внешних факторов.

\section{ЛИТЕРАТУРА}

1. Трусова А.Ю. Многомерный статистический анализ в интернет-маркетинге // Вестник Самарского университета. Экономика и управление. 2018. Т. 9. № 1. C. 68-75.

2. Riazoshams H., Midi H., Ghilagaber G. Robust nonlinear regression: with applications using R. Hoboken: Wiley; 2019.

3. Averkin A.N., Yarushev S.A. Hybrid Approach for Time Series Forecasting Based on ANFIS and Fuzzy Cognitive Maps // Proceedings of the 20th IEEE International Conference on Soft Computing and Measurements (SCM). 2017. PP. 379-381.

4. Подвесовский А.Г., Исаев Р.А. Идентификация структуры и параметров нечетких когнитивных моделей: экспертные и статистические методы // International Journal of Open Information Technologies. 2019. Т. 7. № 6. С. 35-61.

5. Борисов В.В., Луферов В.С. Метод многомерного анализа и прогнозирования состояния сложных систем и процессов на основе нечетких когнитивных темпоральных моделей // Системы управления, связи и безопасности. 2020. № 2. C. 1-23. D01: 10.24411/2410-9916-2020-10201.

6. Klimenko V.V., Klimenko A.V., Tereshin A.G., Mitrova T.A. Impact of Climate Changes on the Regional Energy Balances and Energy Exports from Russia // Thermal Engineering. 2019. Vol. 66. № 1. P. 3-15.

7. Стефанцов А.Г., Бобряков А.В., Борисов В.В., Луферов В.С. Система нечеткого когнитивного анализа и моделирования системной динамики // XXI Международная научная конференция: Проблемы управления и моделирования в сложных системах. 2019. Том 2. с. 312-316.

8. Елисеева И.И. Курышева С.В. Костеева Т.В. Бабаева И.В. Михайлов Б.А. Эконометрика.— М.: Финансы и статистика, 2007.— 192 с. 